\title{
Optimum Access Analysis of Collaborative Spectrum Sensing in Cognitive Radio Network using MRC
}

\author{
Risala Tasin Khan \\ Institute of Information Technology \\ Jahangirnagar University \\ Savar, Bangladesh \\ Shakila Zaman \\ Institute of Information Technology \\ Jahangirnagar University \\ Savar, Bangladesh
}

\author{
Md. Imdadul Islam \\ Computer Science and Engineering \\ Jahangirnagar University \\ Savar, Bangladesh
}

\author{
M. R. Amin \\ Electronics and Communication Engineering \\ East West University \\ Dhaka, Bangladesh
}

\begin{abstract}
The performance of cognitive radio network mainly depends on the finest sensing of the presence or absence of Primary User (PU). The throughput of a Secondary User (SU) can be reduced because of the false detection of $P U$ which causes an SU from its transmission opportunity. The factorization of the probability of correct decision is a really hard job when the special false alarm is incorporated into it. Previous works focus on collaborative sensing on the normal environment. In this paper, we have proposed a collaborative sensing method in Cognitive radio network for optimal access of PU licensed band by SU. It is shown performance analysis of energy detection through different cognitive users and conducts a clear comparison between local and collaborative sensing.In this paper, the maximal ratio combining diversity technique with energy detection has been employed to reduce the false alarm probability in the collaborative environment. The simulation result showssignificant reduction of the probability of misdetection with increasing in the number of collaborative users.We also analyze that MRC scheme exhibits the best detection performance in collaborative environment.
\end{abstract}

Keywords-Fusion center; Local energy detection; Maximum
Ratio Combining; Spectrum Sensing;Receiver Operating Characteristics

\section{INTRODUCTION}

Day by bay spectrum demand is increasing fast with the rapid growth of a new high data rate and wireless devices. Since frequency allocation is fixed and the users do not use the spectrum all time, so it introduces significant underutilization of the available frequency. Cognitive Radio (CR) becomes as a solution to this scarcity by providing more utilization of the spectrum resources which is capable to fulfilling the demand of to be available anyplace, anytime, when needed [1]. CR is an adaptive and smart system that can automatically detect the hidden spectrum hole and provide unused licensed spectrum to the cognitive user by dynamic spectrum sharing. In Cognitive Radio Network (CRN), Primary User (PU) denotes as authorized user who uses the licensed frequency and has higher priority to access the specified spectrum and Secondary User (SU) denotes as unlicensed user who is responsible for sensing the movement of PU's and use the spectrum when PU is in stationary mode.

One of the most significant components of CR is reliable spectrum sensing. Spectrum sensing is the process of discovering unused spectrum which is allocated to PU and make awareness about the existence of PU. Due to shadowing and multipath fading, this is a great challenge to execute spectrum sensing in the hidden terminal and know the status of an instantaneous spectrum. The performance of a good CRN exclusively depends on how accurately the SU can sense the existence or nonexistenceof a PU. Various traditional techniques have been used to implement the spectrum sensing such as matched filter, cyclostationary detection, energy-based detection algorithm which are discussed in [2] and [3]. Energy detection is the most popular and general sensing method due to its less implementation complexity and superiorvelocity which is also known as semi-blind detection method [4]. In this method, correctly threshold value selection is more significant to measure the performance. Since the one or more sensing parameters are unknown in the dynamically changing environment, recent studies [5]-[7]have focused on improving the performance of the detection method. In this paper, we have used noise level estimation to choose significant threshold value to meet constant false alarm rate. For quick and reliable spectrum detection and reduce false alarm, Collaborative Spectrum Sensing (CSS) has been introduced [8]-[11]The purpose of collaborative spectrum sensing induces new design and optimization challenges, such as transmission delay, security risk and energy consumption [12]-[15]. In CSS, secondary users send their local sensing information to the Fusion Center (FC). Then FC fuses the received signal information to decide about absence or presence of PU [16]. In [17], different data fusion scheme is used to optimize the detection performance in FC. At FC different diversity scheme can be implemented to make the final decision such as Maximum Ratio Combining (MRC), Selection Combining (SC) and Square-Law Combining (SLC) [18]. This paper includes energy detection in CSS with Maximum Ratio Combining (MRC) scheme. Maximum ratio combining diversity technique is used to analysis best possible 
access in collaborative environment. Under MRC scheme, multiple cognitive users received the sensing result and send to the FC using data fusion, where the data from multiple cognitive radios are combined by MRC linear combiner. Then an energy detection is used to dealings the MRC combiner output. In [17], Collaborative spectrum sensing is used to verify the efficiency of detecting spectrum holes by different combing scheme.

The goal of this paper is the analysis of the optimum energy detection based on the different parameter in local and collaborative spectrum sensing with MRC using traditional energy detection.The rest of this paper is structured as follows. Preliminary models of local and collaborative energy detection are discussed in Section II. The comprehensive system architecture of collaborative sensing with MRC is also discussed in section II. In section III, simulation parameter and results are given to analysis the optimum spectrum sensing in CRN. Finally, we conclude this paper in section IV.

\section{SYSTEM MODEL}

\section{A. Local energy detection for CR users}

In the case of PU's information is unknown in the Cognitive radio network, most popular PU's detection method is Energy detection. By following fig 1 for known time interval a bandpass filter is used to select collected frequency and bandwidth for energy detection method. SNR is estimated by using channel SNR estimator. Then energy of received signal is measured by magnitude squaring device with an integrator. Measured test statistics is compared with a predefined threshold $\tau$ to produce information about the existence of the Primary user. Spectrum sensing using energy detection is formulated with two hypothesis test also known as binary signal detection. If there is no primary user then hypothesis result produce $H_{0}$, otherwise it produces $H_{1}$ that indicatethe presence of Primary user.

Mathematically the two hypothesis-testing can be formulated as,

$$
x_{k}[t]= \begin{cases}w_{k}[t] ; & H_{0} \\ \alpha_{k} e^{j \theta_{k}}[t]+w_{k}[t] ; & H_{1}\end{cases}
$$

Where $x_{k}[t]$ denoted as received signal at $k^{\text {th }}$ Secondary user with $k=0,1,2,3 \ldots . . . N r$ which is independently and identically distributed [19], $\alpha_{k} e^{j \theta_{k}}$ is the channel gain between PU and SU, $s[t]$ is the PU's transmitted signal that follows the Gaussian random process with zero mean and variance $\sigma_{s}^{2}$ and $w_{k}[t]$ denotes the white noise.

To determine the efficient performance of energy sensing method the test estimation is defined as,

$$
T(x)=\frac{1}{M} \sum_{k=0}^{M} x_{k}[t]^{2} ;
$$

Where $M$ is the size of observation vector and $T(x)$ is text statistics.

Two important detection probability parameters are used to measure the performance of any detection method that is $P_{D}$ and $P_{F A} . P_{D}$ indicates the probability of detecting the existence of the signal of PU on the required frequency when it is actually present. Since PU is surrounded by various interference, $P_{D}$ should be as large as possible to make the correct decision. This detection condition can be written as,

$$
P_{D}=\operatorname{Pr}\left(\text { Signalisdetected } \mid H_{1}\right)=\operatorname{Pr}\left(T(x)>\tau \mid H_{1}\right)
$$

$P_{F A}$ is the probability of choosing $H_{1}$ but $H_{0}$ is true, that means CR's decides that primary user is detected as on mood butactually there is no primary user. To utilize more transmission opportunities of unutilized spectrum $P_{F A}$ should be as small as possible. That can be expressed as,

$$
P_{F A}=\operatorname{Pr}\left(\text { Signalisdetected } \mid H_{0}\right)=\operatorname{Pr}\left(T(x)>\tau \mid H_{0}\right)
$$

Another important parameter is used to determine the performance of Cognitive user that is the probability of misdetection $P_{M D}$. This condition occurs when a cognitive user (SU) choose $H_{0}$ but $H_{1}$ is true. In this case performance of SU detection will decrease and it can be formulated as,

$$
P_{M D}=\operatorname{Pr}(\text { Signalisnotdetected } \mid 1)
$$

The existence decision of a PU can be estimated by comparing the decision matrix $T(x)$ and a fixed predefine threshold $\tau$. It is very important to identify required threshold value that may change based upon environment condition that is related to the distance between PU and SU.

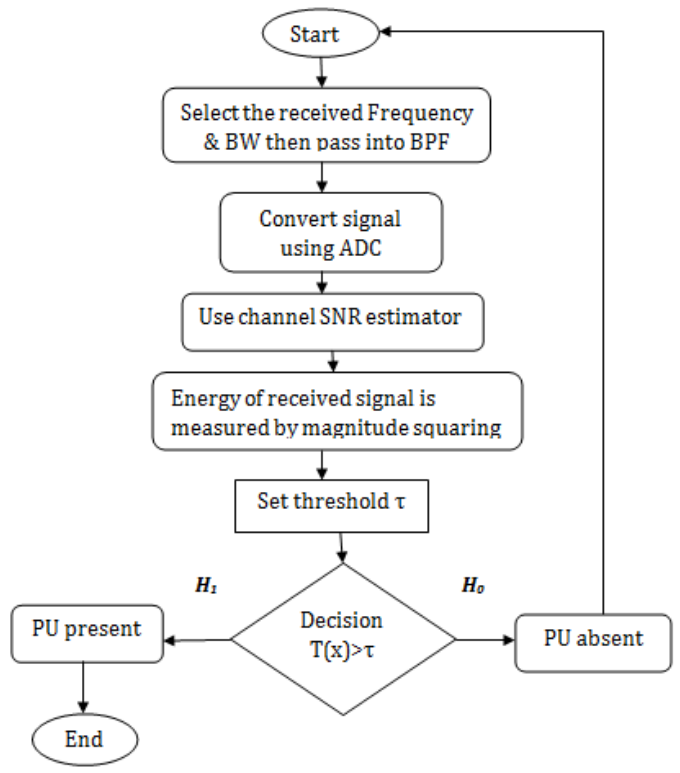

Fig. 1. Local Energy Detection for Cognitive user

Threshold value can be estimated by given equation that is based upon signal noise, detected signal energy, the size of observation sample and noise variance. Since the distance between Cognitive user and primary user changes, it is difficult to estimate signal energy. So threshold value is chosen to meet constant false alarm rate using noise level estimation.

That is calculated by given equation [20],

$$
\tau=\sqrt{\frac{\sigma_{S}^{2}}{M} Q^{-1}\left(P_{F A}\right)}(2)
$$

where,

$\tau=$ threshold,

$\sigma_{s}^{2}=$ noise Variance,

$M=$ size of observation vector or sample. 
To estimate probability of correct detection we can choose the required threshold value from equation (2) and the probability of detection can be formulated as [21],

$$
P_{D}=Q\left(\left(\frac{\tau}{\sigma_{s}^{2}}-\gamma-1\right) \sqrt{\frac{\delta_{t} f_{s}}{2 \gamma+1}} \cdot Q^{-1}\left(P_{F A}\right)\right)
$$

where,

$f_{s}=$ sampling frequency,

$Q()=$. complementary distribution function of standard

Gaussian,

$\delta_{t}=$ sensing time or duration.

Then $P_{F A}$ is given by,

$$
P_{F A}=Q\left(\left(\frac{\tau}{\sigma_{s}^{2}}-1\right) \sqrt{\delta_{t} f_{s}}\right)(4)
$$

The probability of misdetection is complement of $P_{D}$ which can be formulated as,

$$
P_{M D}=1-P_{D}(5)
$$

From [21] for a target false alarm $P_{F A}$, misdetection probability is given by,

$$
\begin{aligned}
& P_{M D}=1-P_{D} \\
= & 1-Q\left(\frac{1}{\sqrt{2 \gamma+1}}\left(Q^{-1}\left(P_{F A}\right)-\sqrt{\delta_{t} f_{s} \gamma}\right)\right)
\end{aligned}
$$

Therefore, $P_{F A}$ is related to targeted detection probability which is formulated as [21],

$$
P_{F A}=Q\left(\sqrt{2 \gamma+1}\left(Q^{-1}\left(1-P_{M D}\right)-\sqrt{\delta_{t} f_{s} \gamma}\right)\right)(7)
$$

\section{B. Collborative Spectrum sensing Including MRC scheme}

a) Formulation:To decreases false alarm probability, misdetection and also mitigate the hidden problem in the CRN, collaborative spectrum sensing has been introduced. In this case, multiple Cognitive users sense the spectrum band collaboratively. According to fig 2, data fusion method is used in all cognitive radio users to sense their spectrum independently but they do not make any decision to get the opportunity to transmit. All individual node transmit their sensing information to a central Fusion Centre (FC) using local sensing method. Then FC makes the final decision whether the SU transmit or not using the information of PU present or absent.

If we consider a CRN with n number of user where $n=1$, 2 , 3........Nr, the probability of collaborative detection is formulated as [22],

$$
Q_{D}=1-\left(1-P_{D}\right)^{n}
$$

Then collaborative false alarm probability is given by,

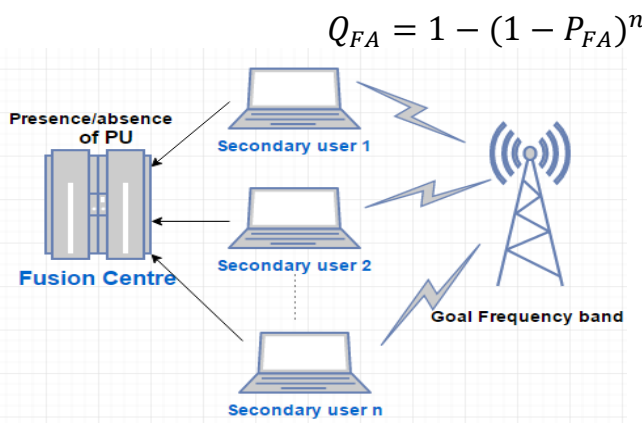

Fig. 2. State diagram of collaborative spectrumsensing
In FC, various combining techniques are used to combine the collected sensing information that comes from $\mathrm{n}$ number of independent cognitive users. In this paper, we have considereda case where FC make a decision whether the PU is absent or present using Maximum Ratio Combining scheme. According to fig 3, multiple cognitive radio users directly forward their sensing decision to the FC where the collected information is combined by an MRC scheme using linear combiner.

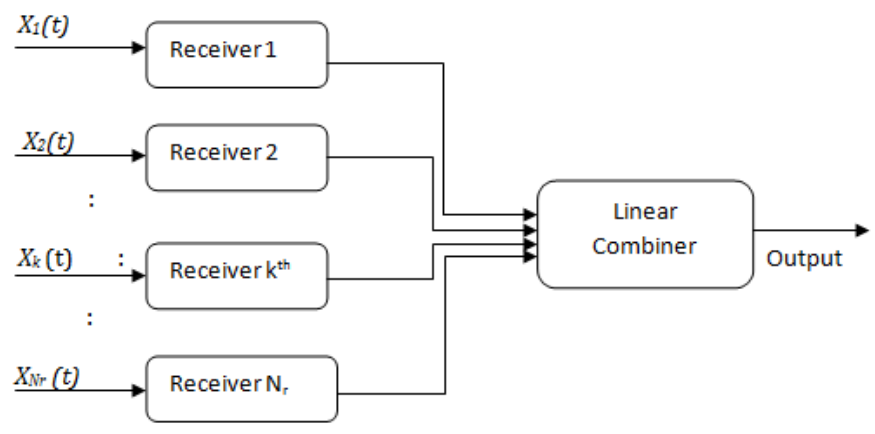

Fig. 3. Block diagram of Maximum ratio combining using $N_{r}$ receiving antenna

Using Complex envelop of receive signal for $k^{\text {th }}$ branches in MRC technique the corresponding received linear combined signal is calculated as [23],

$$
\begin{array}{r}
\tilde{y}[t]=\sum_{k=1}^{N_{r}} \xi_{k} \tilde{x}_{k}[t] \\
=\sum_{k=1}^{N_{r}} \xi_{k}\left[\alpha_{k} e^{j \theta_{k}}[t]+\widetilde{w}_{k}[t]\right]
\end{array}
$$

where,$$
\alpha_{k} e^{j \theta_{k}}=\text { complex factor or channel gain in fading, }
$$$$
\xi_{k}=\text { complex weighted factor for each channel that }
$$$$
\text { characterize the linear combiner, }
$$$$
\sum_{k=1}^{N_{r}} \alpha_{k} e^{j \theta_{k}}[t]=\text { complex envelop of output signal, }
$$$$
\sum_{k=1}^{N_{r}} \widetilde{w}_{k}[t]=\text { complex envelop of output noise. }
$$

In this case, two hypothesisis mathematically formulated

$$
\text { as, } \tilde{x}_{k}[t]= \begin{cases}\sum_{k=1}^{N_{r}} \widetilde{w}_{k}[t] ; & H_{0} \\ \sum_{k=1}^{N_{r}} \alpha_{k} e^{j \theta_{k}}[t]+\widetilde{w}_{k}[t] ; & H_{1}\end{cases}
$$

Therefore, the MRC technique produces an instantaneous output SNR that is denoted as $\gamma_{m r c}$ which maximizes the detectionprobability in collaborative spectrum sensing manner. Thatproduce by summarizing all individual users instantaneous SNR using linear combiner and given by [23],

and,

$$
\gamma_{m r c}=\sum_{k=1}^{N_{r}} \gamma_{k}
$$

$$
\gamma_{k}=\frac{E_{S}}{N_{0}} \alpha_{k}^{2}
$$

where,

$\gamma_{k}=$ instantaneous SNR for the individual $k^{t h}$ receiver where

$k=1,2,3 \ldots \ldots . . N r$,

$E_{s}=$ symbol energy,

$N_{0}=$ one-sided noise spectral density. 
Then the targeted collaborative detection and false alarm probability under MRC combiner scheme can be given by, $P_{M D}=1-\left(1-Q\left(\frac{1}{\sqrt{2 \gamma_{m r c}+1}}\left(Q^{-1}\left(P_{F A}\right)-\sqrt{\delta_{t} f_{s} \gamma_{m r c}}\right)\right)\right)^{n}$

$P_{F A}=1-\left(1-Q\left(\sqrt{2 \gamma_{m r c}+1}\left(Q^{-1}\left(1-P_{M D}\right)-\right.\right.\right.$ $\left.\left.\sqrt{\delta_{t} f_{s} \gamma_{m r c}}\right)\right)^{n}(14)$

\section{b) System Architecture:}

In fig 4, a flowchart is proposed that shows the energy detection steps with MRC scheme in collaborative sensing manner. In this technique, each SU sends their local information to FC and FC calculate the energy by linear combiner and also calculate weighted and fading complex factor then produce $\gamma_{m r c}$ using equation(11). Finally, calculate test statistics and compare with predefined threshold $\tau$, then make a decision about the existence of PU. MRC system can improve the performance and the bandwidth efficiency in CRN. In this paper, a new algorithm, maximum ratio combining with energy detection is proposed for collaborative sensing environment where itshows step by step procedure to improve the performance over frequency sensing channel.

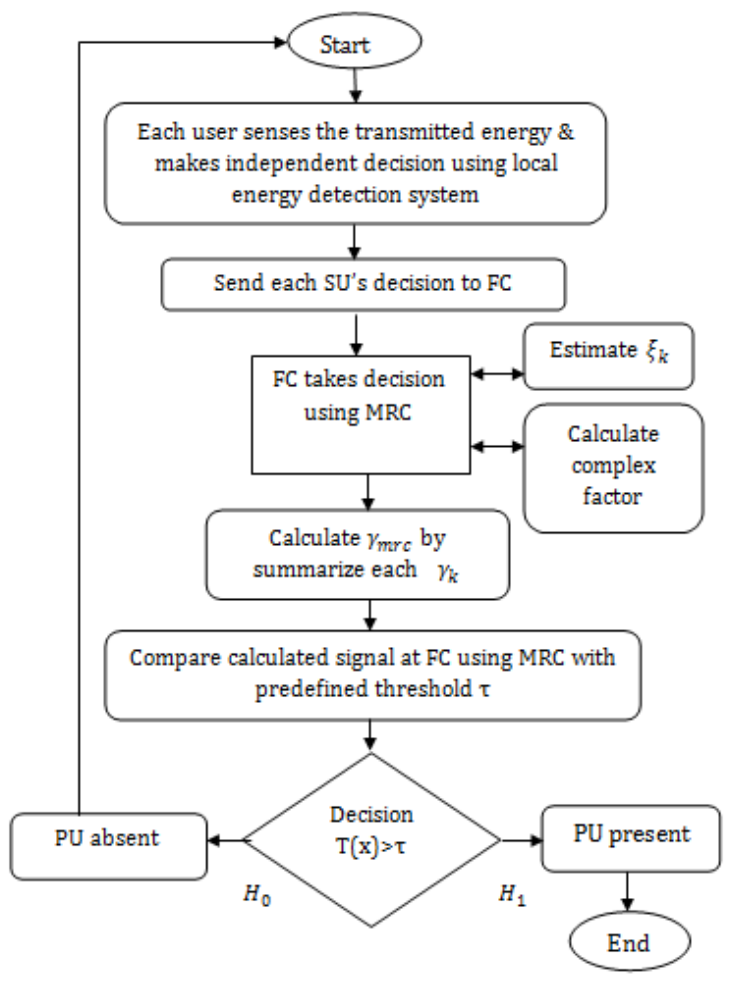

Fig. 4. Collaborative Energy detection using MRC

\author{
Step 5: Select thenumber of users $(n)$ as thecollaborative \\ sample.
}

Step 6: Each CR's Report the sensing information $\left(H_{1}\right.$ or $\left.H_{0}\right)$ to FC.

Step 7: FC takesthe final opportunistic decision using MRC.

Step 8: Calculate weighted factor $\left(\xi_{k}\right)$ for each SU's.

Step 9: Calculate Complex factor $\alpha_{k} e^{j \theta_{k}}$.

Step 10: Calculate $\gamma_{m r c}$ using equation (11).

Step 11: Compute test statistics $T(x)$.

Step 12: Compare $T(x)$ with $\tau$ and return any one of two hypothesis decision.

Step 13: Compute $P_{D}, P_{F A}$ and $P_{M D}$ using required parameters. Step 14: Calculate $Q_{D}$ and $Q_{F A}$ for estimate Collaborative sensing efficiency.

\section{SimUlation AND RESUlt}

\section{A. Simulation parameters}

Table I include the simulation parameters followed by the above scheme. To calculate local detection probability, false alarm probability and collaborative detection with MRC following parameters are considered.

TABLE I. PARAMETERS For CALCUlating PD, PMA AND PFAFOR LOCAL AND COLLABORATIVE ENVIRONMENT

\begin{tabular}{|c|c|c|}
\hline Parameter & Description & Value \\
\hline$\tau$ & Threshold & $.002-.04$ \\
\hline$\gamma$ & Instantaneous SNR & $2-15 \mathrm{db}$ \\
\hline$\delta_{t}$ & Sensing time & $50-150 \mathrm{~ms}$ \\
\hline$f_{s}$ & Sampling frequency & $10-400 \mathrm{~Hz}$ \\
\hline$\sigma_{S}$ & Variance & .001 \\
\hline$n$ & Number of user & $1-30$ \\
\hline$M$ & Size of sampling vector & $20-60$ \\
\hline$P_{F A}$ & False alarm probability & $.001-.5$ \\
\hline
\end{tabular}

\section{B. Simulation Results}

To identify the tradeoff between the probability of detection and probability of false alarm, Receiver Operating Characteristics(ROC) analysis has been used. This section provides simulation and analytical result to verify and compare the ROC curves in different scenarios. All figures show that theoretical results are closely meet with simulation result. Therefore, maximum confidence level is achieved. At first, we show the performance and tradeoff between probability of detection and false alarm for non-cooperative sensing environment which is very important to compare the sensing efficiency in a collaborative manner. For a perfect reporting channel, the basic requirement is to identify the threshold. In this paper, the threshold selection is carried out by considering present conditions of noise level using constant false alarm rate method from equation (2).

\footnotetext{
Algorithm 1Steps to calculate Collaborative Spectrum Sensing efficiency with MRC

Step 1: Each CR's collect signal independently and pass through Bandpass Filter (BPF).

Step 2: Channel SNR $(\gamma)$ estimation.

Step 3: Set $\tau$ from equation (2) that consider constant false alarm using noise level estimation.

Step 4: Estimate $\sigma_{s}^{2}$.
} 


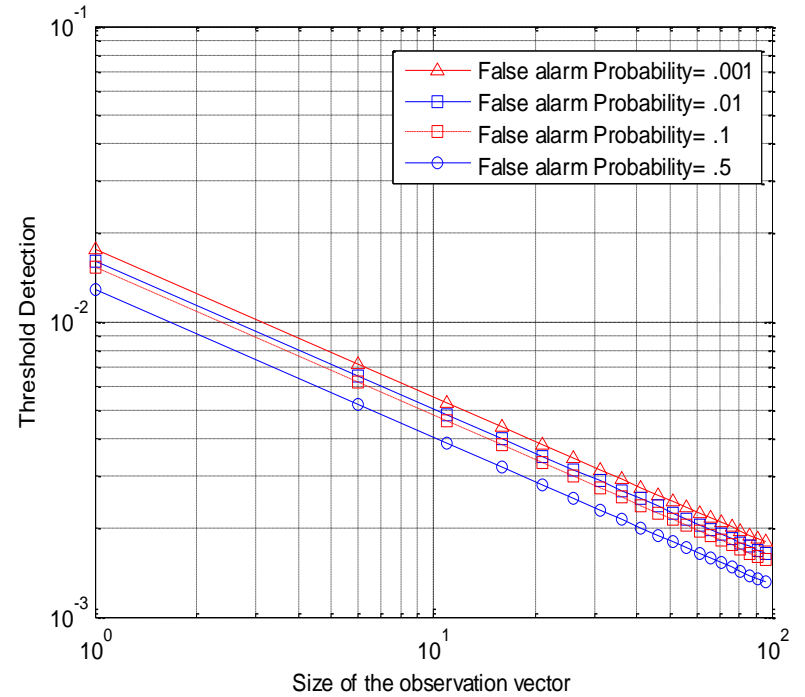

Fig. 5. Complementary ROC curves of threshold detection over size of observation vector for constant false alarm rate

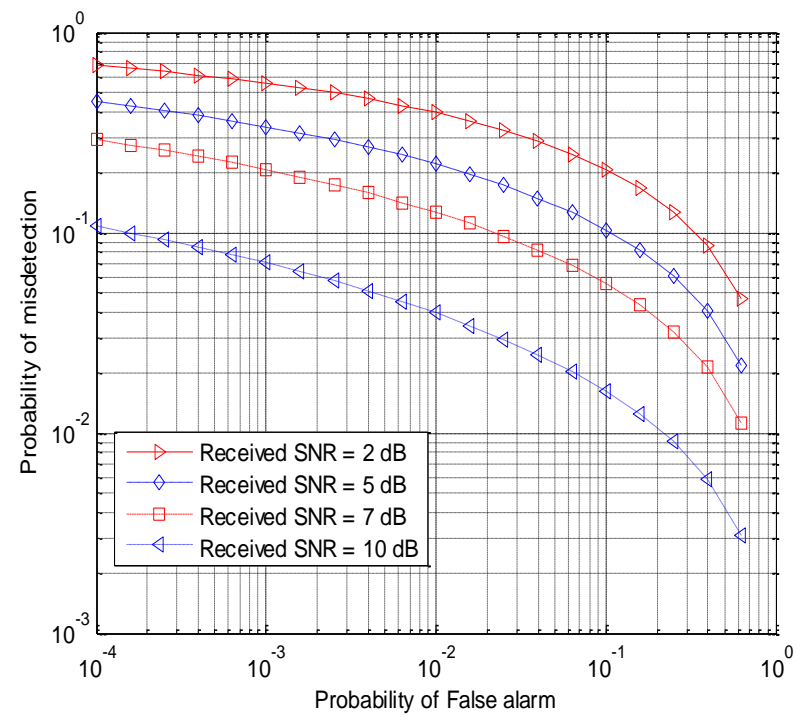

Fig. 6. Complementary ROC curves of energy detection over AWGN

Fig 5 shows ROC curves to identify perfect threshold value for different constant false alarm rate. Fig 6 shows the effect of probability of false alarm on misdetection where each user sees different SNR. We observe from this figure that for large SNR the probability of misdetection over false alarm will decrease. It also shows that energy detection works better for higher SNR. Fig 7 shows the performance of an energy detector for fixed false alarm rate which varies with frequency. From Fig 7, it is observed that when the $\mathrm{P}_{\mathrm{FA}}$ decreases, the $\mathrm{P}_{\mathrm{MD}}$ also gradually decreases. For low false alarm rate probability of misdetection remain low. Fig 8 demonstrates that for a large spectrum sensing period the performance of energy detection increases with less approximate error.

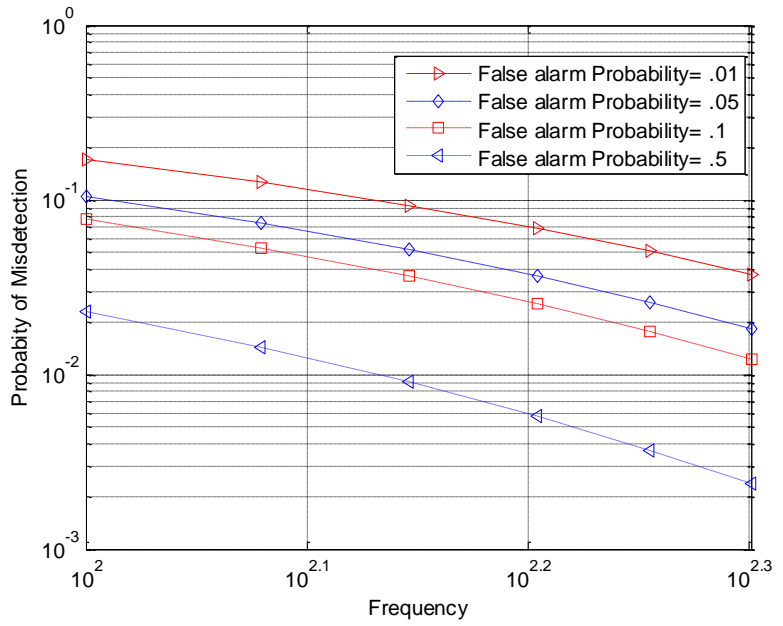

Fig. 7. Variation of the probability if misdetection against frequency for fixed false alarm rate

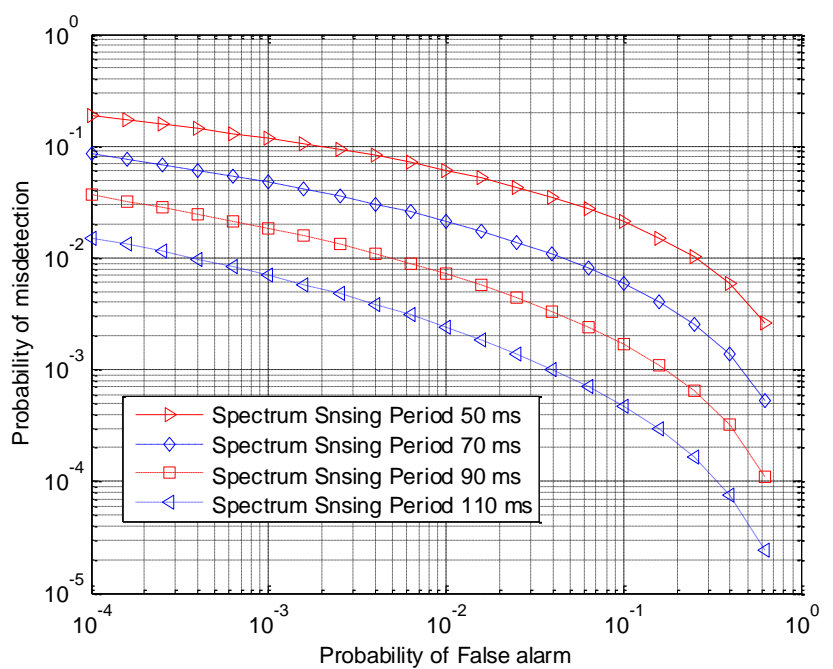

Fig. 8. ROC curve for the probability of misdetection VS probability of false alarm for various sensing period

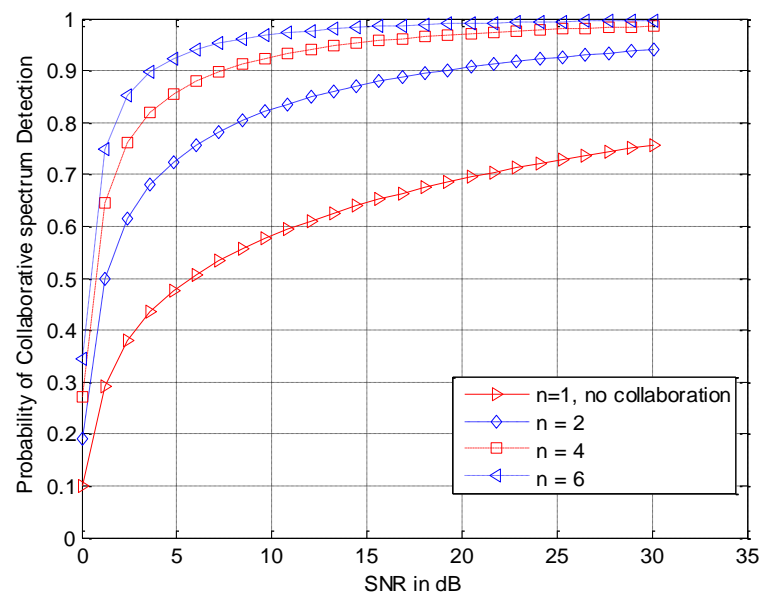

Fig. 9. Variation of probability of detection against SNR for different number of receiving antenna 
Now we discuss about the performance of collaborative spectrum sensing cases. In these following simulations, it has been observed that collaborative spectrum sensing works better than non-collaborative environment. Figs 9-11 manifest that the probabilities of collaborative detection will decreases when either the number of collaborative user $n$ or SNR decreases. Large number ofcollaborative user produces better performance with less misdetection in FC. Fig 10 shows the effect of number of collaborative users on the probability of collaborative detection. It has been observed for the figure that the collaborative detection rises with the sensing time. Fig 12 shows the ROC curve of comparison between normal collaborative (without diversity scheme) and collaborative detection using MRC diversity. We observe from this figure that MRC scheme exhibits the best detection performance with energy detection in collaborative environment though it requires channel state information withcomplex factor discussed in section II.

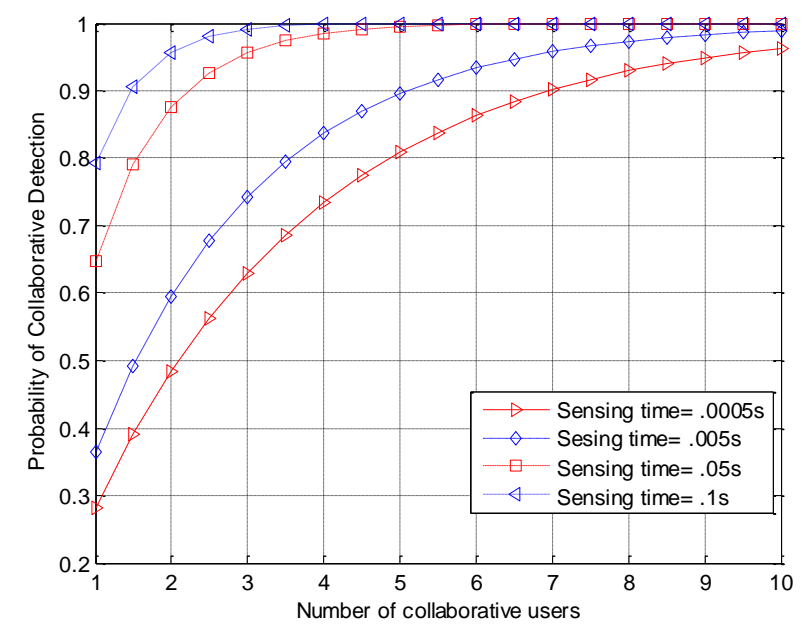

Fig. 10. ROC curve for energy detection VS. number of users for different sensing period in collaborative manner

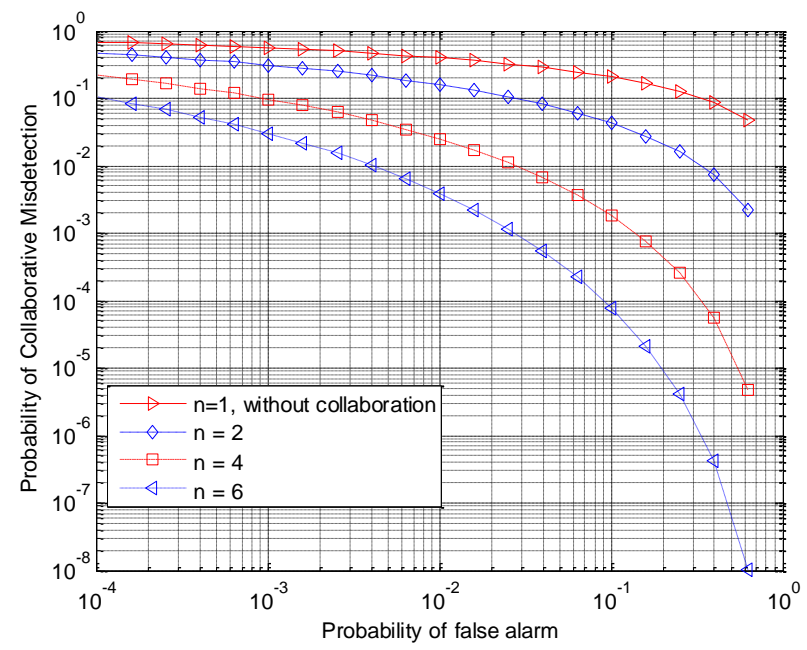

Fig. 11. Complementary ROC curves for collaborative energy detection against false alarm for different number of receiving antenna

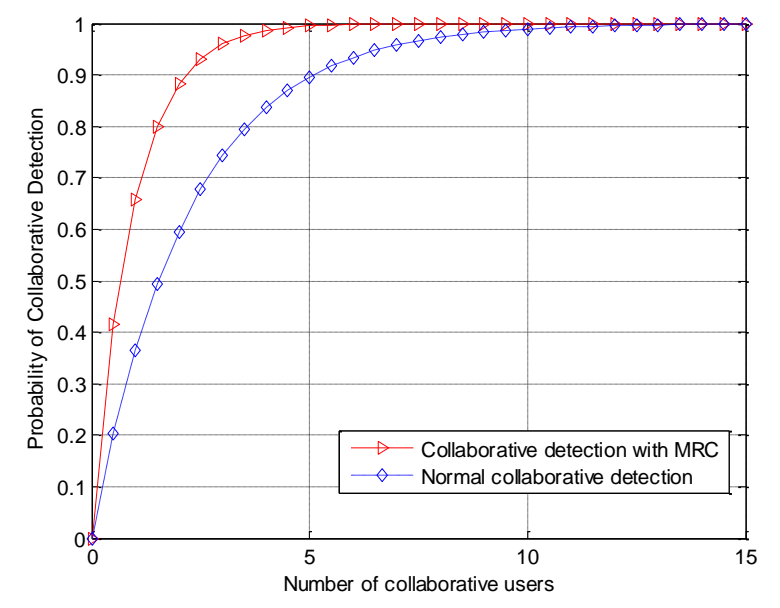

Fig. 12. Complementary ROC curve of collaborative detection with MRC

\section{CONCLUSION}

In this paper spectrum sensing concepts are re-evaluated with collaborative sensing using MRC by considering different dimensions of the spectrum space. We have used energydetection method to sensing unused spectrum. In this method, probability of correct decision completely depends upon appropriate value of threshold. In our paper, an adaptive thresholdalgorithm is used to select suitable threshold value in vigorously changing environment. We have focused on optimum spectrum sensing in cognitive radio network based on differentrequired parameters. This paper proposes a new architecture in collaborative spectrum sensing with MRC diversity. This System provides efficient spectrum sensing and consequently leads to enhanced CR performance. From the simulation results, it is observed that there is significant reduction of the probability of misdetection with increasing in the number of collaborative user.

\section{REFERENCES}

[1] S haykin, "Cognitive radio: Brain-empowered wireless communication", IEEE Journal Selected Areas in Communications, vol.23, no.2, pp.201-202, Feb.2005.

[2] J. G. Proakis, "Digital communications" ed: McGraw-Hill, New York, 1995.

[3] Aamir Zeb Shaikh, Dr. Talat Altaf, "Collaborative spectrum sensing under suburban environments", (IJACSA) International Journal of Advanced Computer Science and Applications, Vol. 4, No.7, 2013.

[4] Y. Zeng, et al., "A review on spectrum sensing for cognitive radio: challenges and solutions," EURASIP Journal on Advances in Signal Processing, vol. 2010, pp. 2, 2010.

[5] Daniela Mercedes Martnez Plataa, ngel Gabriel Andrade Retiga, "Evaluation of energy detection for spectrum sensing based on dynamicselection of detection threshold", International Meeting of Electrical Engineering Research, ENIINVIE-2012.

[6] Deepak R. Joshi, Dimitrie C. Popescu and Octavia A. Dobre, "Adaptive spectrum sensing with noise variance estimation for dynamic cognitive radio systems", IEEE, 2010.

[7] Adeel Ahmed, Yim Fun Yu,James M.Nora , " Noise Variance estimationfor spectrum sensing in cognitive radio" AASRI Conference on circuitsand signal processing, 2014.

[8] Y. Liang, L. Lai, and J. Halloran, "Distributed algorithm for collaborative detection in cognitive radio networks", Communication Control and Computing, pp. 394 -399, 2009. 
[9] H. Li, H. Dai, and C. Li, "Collaborative quickest spectrum sensing via random broadcast in cognitive radio systems", IEEE Global Telecommunications Conference, pp. 1-6, 2009.

[10] H. Teng-Cheng, W. Tsang-Yi, and H. Y.-W. Peter, "Collaborative change detection for efficient spectrum sensing in cognitive radio networks", IEEE Vehicular Technology Conference, pp. 1-5, 2010.

[11] H. Li, M. Junfei, X. Fangmin, L. ShuRong, and Z. Zheng., "Optimization of collaborative spectrum sensing for cognitive radio", NetworkingSensing and Control, pp. 1730-1733, 2008.

[12] Yue Wang, Justin P. Coon, Angela Doufexi, ”Energy-efficient spectrum sensing and access for cognitive radio networks. Vehicular Technology", IEEE Transactions on, Vol.61(2), pp. 906-912, 2012.

[13] Mesodiakaki A., Adelantado F., Alonso L., and Verikoukis C., "Energy effi- ciency analysis of secondary networks in cognitive radio systems" In Communications (ICC), IEEE International Conference on, (pp. 4115-4119). IEEE, June 2013.

[14] Adelantado F., and Verikoukis C., "Detection of malicious users in cognitive radio ad hoc networks: A non-parametric statistical approach" Ad Hoc Networks, Vol.11(8), pp.2367-2380, 2013.

[15] Wang, W., Wu, K., Luo, H., Yu, G., and Zhang, Z., "Sensing error aware delay-optimal channel allocation scheme for cognitive radio networks", Telecommunication Systems, Vol. 52(4), pp. 1895-1904, 2013.

[16] D.cabric, S.Mishra, R.Brodersen , "Implementation issues in spectrum sensing for cognitive radios" in: Proc. Of Asilomar Conf. on Signals, System and Computers, vol.1, pp.772-777, 2004.
[17] D. Teguig, B. Scheers and V. Le Nir, "Data fusion schemes for cooperative spectrum sensing in cognitive radio networks", Communications and Information Systems Conference (MCC), Military, IEEE 8-9 , pp:1 7 Print ISBN: 978-1-4673-1422-0, Oct.2012.

[18] F. F. Digham, M. S. Alouini, and M. K. Simon, "On the energy detection of unknown signals over fading channels", IEEE Trans. Commun., vol. 55, no. 1, pp. 2124, Jan. 2007.

[19] E. Visotsky, et al., "On collaborative detection of TV transmissions in support of dynamic spectrum sharing", in New Frontiers in Dynamic Spectrum Access Networks, First IEEE International Symposium on, pp. 338-345,2005.

[20] Deep Raman and N.P.Sing, "An Algorithm for spectrum sensing in Cognitive Radio under noise uncertainty", International journal of Future Generation communication and Networking, Vol.7, No. 3, pp. 61-68, 2014.

[21] Ahmed El Shafie, "Optimal Spectrum Access for Cognitive Radios", Wireless Intelligent Networks Center (WINC), Nile University, Giza, Egypt. 24, arXiv:1208.4508v5 [cs.IT], 24 Mar 2013.

[22] Xiaoge Huang, Ning Han, Guanbo Zheng, Sunghwan Sohn, Jaemoung Kim, "Weighted-Collaborative Spectrum Sensing in Cognitive Radio" ,Communications and Networking in China, CHINACOM Vol.07. Second International Conference on, 2007.

[23] Simon O. Haykin, Michael Moher, "Modern Wireless Communications", ISBN-10: 0130224723. 